\title{
Structure of Magnetically Ordered Si:Mn
}

\author{
J. Bak-Misiuk, , a , E. Dynowska ${ }^{1, b}$, P. Romanowski ${ }^{1, ~ c}$, A. Shalimov ${ }^{1, d}$, \\ A. Misiuk ${ }^{2, ~ e}$, S. Kret ${ }^{1, f}$, P. Dluzewski ${ }^{1, g}$, J. Domagala ${ }^{1, h}$, W. Caliebe ${ }^{3, \text { i }}$, \\ J. Dabrowski ${ }^{1, j}$ and M. Prujszczyk ${ }^{2, e}$ \\ ${ }^{1}$ Institute of Physics, PAS, Al. Lotnikow 32/46, 02-668 Warsaw, Poland \\ ${ }^{2}$ Institute of Electron Technology, Al. Lotnikow 46, 02-668 Warsaw, Poland \\ ${ }^{3}$ HASYLAB at DESY, Notkerstrasse 85, D-22603, Hamburg, Germany \\ abakmi@ifpan.edu.pl, ${ }^{b} d y n o w @ i f p a n . e d u . p l,{ }^{c}$ romeck@ifpan.edu.pl, \\ d ${ }^{d}$ shalim@ifpan.edu.pl, ${ }^{e}$ misiuk@ite.waw.pl, ${ }^{f}$ kret @ifpan.edu.pl, ${ }^{9}$ dluzew@ifpan.edu.pl, \\ hdomag@ifpan.edu.pl, 'wolfgang.caliebe@desy.de, 'dabrow@ifpan.edu.pl
}

Keywords: Silicon, manganese, implantation, annealing, high pressure, structure, magnetic ordering.

\begin{abstract}
The structure studies of single crystalline silicon implanted at $340 \mathrm{~K}$ or $610 \mathrm{~K}$ with $\mathrm{Mn}^{+}$ ions (Si:Mn) and subsequently processed under atmospheric and enhanced hydrostatic pressure at up to $1270 \mathrm{~K}$ are reported. The defect structure was determined by an analysis of $\mathrm{X}$-ray diffuse scattering around the 004 reciprocal lattice point and by electron microscopy. High resolution X-ray diffraction techniques based on the conventional source of radiation were used for this purpose. The crystal structure of $\mathrm{Si}: \mathrm{Mn}$ and the $\mathrm{Si}_{1-x} \mathrm{Mn}_{x}$ precipitates in the implantation disturbed layer were studied by synchrotron radiation diffraction in the grazing incidence geometry. Processing of Si:Mn results in crystallization of amorphous $\mathrm{Si}$ within the buried implantation - disturbed layer and in formation of $\mathrm{Mn}_{4} \mathrm{Si}_{7}$ precipitates. Structural changes are dependent both on temperature of the Si substrate at implantation and on processing parameters.
\end{abstract}

\section{Introduction}

In recent years many efforts have been devoted to synthesis and study the ferromagnetic semiconductors because of interesting physical phenomena involved and of the relevance of magnetism for optoelectronic device technology. Availability of suitable materials for device manufacturing is the key to success of spintronics. The natural choice are so called diluted magnetic semiconductors (DMS), i.e. mixed crystals based on classical semiconductors, with a controlled fraction of nonmagnetic cations substituted by magnetic ions [1,2]. It is obvious that room temperature ferromagnetism is necessary for commercial applications.

Although large effort has been done during recent years to prepare room temperature ferromagnetic III-V semiconductors [3], no satisfying material has been fabricated as so far. Either ferromagnetism is observed only at below room temperature (e.g. in GaMnAs and InMnAs [4]) or no ferromagnetic ordering has been detected (e.g. in $\mathrm{GaMnN} \mathrm{[5]).} \mathrm{On} \mathrm{the} \mathrm{other} \mathrm{hand,} \mathrm{it} \mathrm{has} \mathrm{been}$ demonstrated that, during the growth of magnetic III-V semiconductors, ferromagnetic (often at room temperature) precipitates are fairly easily produced, yielding multi-phase materials [6]. Epitaxially grown $\mathrm{Mn}_{x} \mathrm{Si}_{1-x}(x=5$ at. \%) thin film produces a material with anomalous Hall effect around $70 \mathrm{~K}$ which suggests internal magnetization of local Mn spins [7].

Ion implantation has also been utilized to achieve ferromagnetism in semiconductor crystals. Ferromagnetic ordering in silicon implanted with $\mathrm{Mn}^{+}$ions (Si:Mn) has been reported recently; this ordering is evidently related to the structure of Mn-enriched near-surface layer in the implanted material [8]. It has been found that, for Si:Mn produced by implantation with $\mathrm{Mn}^{+}$doses, $D=10^{15}$ - 
$10^{16} \mathrm{~cm}^{-2}$, at energy, $E=300 \mathrm{keV}$, Curie temperature exceeds $400 \mathrm{~K}$ after rapid thermal annealing at 1070 K. Ferromagnetic properties of $\mathrm{Si}: \mathrm{Mn}$ have been attributed to formation of $\mathrm{MnSi}_{1.7}$ [9]. As it has been stated earlier [10,11], not only temperature but also enhanced hydrostatic pressure of ambient $(H P)$ at processing of Si:Mn affect its structural and magnetic properties.

This work deals with the Si:Mn structure as well as with its dependence on temperature during implantation and on parameters of subsequent processing.

\section{Experimental}

Si:Mn was prepared by implantation of single crystalline Si with $160 \mathrm{keV} \mathrm{Mn}{ }^{+}$ions at $T_{s}=340 \mathrm{~K}$ and $610 \mathrm{~K}$ to a dose, $D=1 \times 10^{16} \mathrm{~cm}^{-2}$. Projected range $\left(R_{p}\right)$ of $\mathrm{Mn}^{+}$equals to $140 \pm 50 \mathrm{~nm}$. Si:Mn was processed at temperature $\left(T_{a}\right)$ up to $1270 \mathrm{~K}$ for $1 \mathrm{~h}$ under ambient $\left(10^{5} \mathrm{~Pa}\right)$ and enhanced $\mathrm{Ar}$ hydrostatic pressures up to $1.2 \mathrm{GPa}$.

The defect structure of samples was determined by high resolution X-ray diffraction using the conventional and synchrotron radiation sources (HASYLAB-DESY) as well as by electron microscopy (TEM). Conventional X-ray investigations were carried out using MRD-PHILIPS diffractometer in the double and triple axis configurations. Reciprocal space maps (RSMs) for the (004) reflections were registered for the two perpendicular azimuthal positions in respect to the diffraction plane (the $[110]$ or $[110]$ directions of the sample were perpendicular to it). Simulation of RSMs was performed using the kinematical theory of X-ray diffraction.

The specimens for cross-sectional TEM investigation were prepared by mechanical polishing and etching to a thickness of about $30 \mu \mathrm{m}$. Electron transparency was obtained by ion milling with Ar ions accelerated $4 \mathrm{kV}$ (Linda Technoorg device). Conventional and high resolution TEM investigations were performed using the JEOL 2000EX microscope equipped with the $\mathrm{LaB}_{6}$ electron gun.

The defect structure was determined from the X-ray diffuse scattering data near the Bragg reflection point [12]. Simulation of reciprocal space maps was performed using the X-ray diffraction kinematical theory. The maps of reciprocal space near the 004 reflection have been recorded to study the symmetry of X-ray diffuse scattering (the axes in Figs 1-3 are marked in the $\lambda / 2 \mathrm{~d}$ units, where $\lambda$ is the wavelength and $d-$ the inter-planar distance).

The phase analysis of the near-surface layers was performed using synchrotron radiation in the grazing incidence geometry. In this method a sample is fixed and the angle between the sample surface and X-ray beam is small ( $1^{\circ}$ in our case). The intensity of diffracted beam has been detected by the counter rotating in the plane perpendicular to the sample surface in the large $2 \theta$ angles ( $2 \theta$ scan). The same geometry has been used for investigation of thin near-surface polycrystalline layers.

\section{Results and Discussion}

The reciprocal space maps of Si:Mn implanted at $340 \mathrm{~K}$ and $610 \mathrm{~K}$ are presented in Figs 1 and 2, respectively. The presented reciprocal space maps correspond to such azimuthal position of the sample in respect to the diffraction plane, for which X-ray diffuse scattering is most symmetrical in respect to its maximum intensity.

Strain related to the layered structure is detected for Si:Mn implanted at $340 \mathrm{~K}$ and processed at $610 \mathrm{~K}$; it disappears after annealing at $1270 \mathrm{~K}$. Processing under $H P$ results in the decreased diffuse scattering intensity. This means that the concentration of defects in this case also decreases. From simulation of RSM's it follows that processing at $1270 \mathrm{~K}$ results in a creation of so called week type of defects [13], for example dislocation loops or of stacking faults (Fig. 1c, d). 
(a)

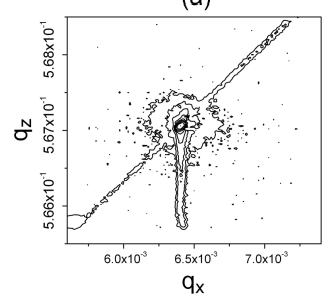

(b)

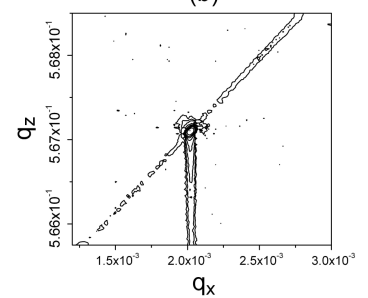

(c)

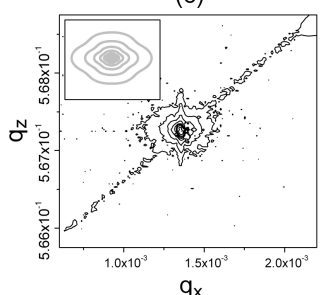

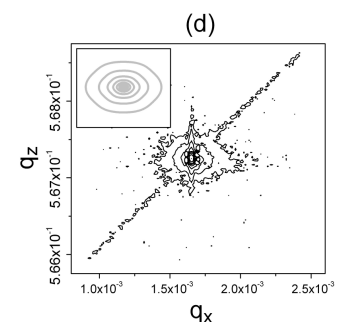

Fig. 1. 004 RSMs of Si:Mn implanted at $340 \mathrm{~K}$ and processed for $1 \mathrm{~h}$ at: (a) $610 \mathrm{~K}$ under $10^{5} \mathrm{~Pa}$; (b) $610 \mathrm{~K}$ under $1.1 \mathrm{GPa}$; (c) $1270 \mathrm{~K}$ under $10^{5} \mathrm{~Pa}$; (d) $1270 \mathrm{~K}$ under $1.1 \mathrm{GPa}$.

(a)

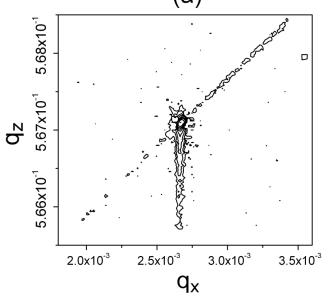

(b)

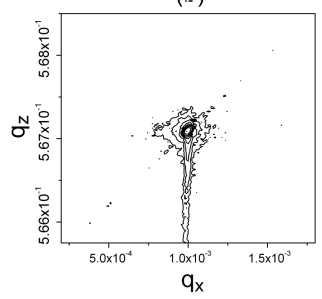

(c)

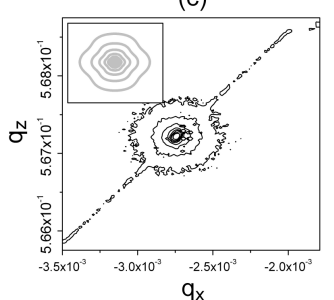

(d)

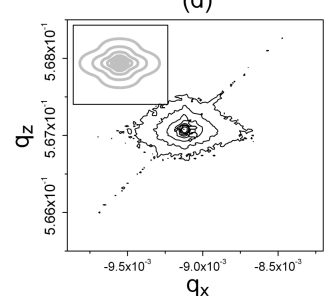

Fig. 2. 004 RSMs of Si:Mn implanted at $610 \mathrm{~K}$ and processed for $1 \mathrm{~h}$ at: (a) $610 \mathrm{~K}$ under $10^{5} \mathrm{~Pa}$; (b) at $610 \mathrm{~K}$ under $1.1 \mathrm{GPa}$; (c) $1270 \mathrm{~K}$ under $10^{5} \mathrm{~Pa}$; (d) $1270 \mathrm{~K}$ under $1.1 \mathrm{GPa}$.

For the samples implanted at $610 \mathrm{~K}$ and processed also at $610 \mathrm{~K}$, the layered structure was detected similarly as in the previous case, but in this case $H P$ results in a more pronounced creation of defects. Processing at $1270 \mathrm{~K}$ results also in a creation of the weak type of defects (similarly as for Si:Mn implanted at $340 \mathrm{~K}$ ).

However, processing at $870 \mathrm{~K}$ results in a creation of precipitates composed of the tetragonal $\mathrm{Si}_{7} \mathrm{Mn}_{4}$ phase and placed within the buried implantation - disturbed layer.

(a)

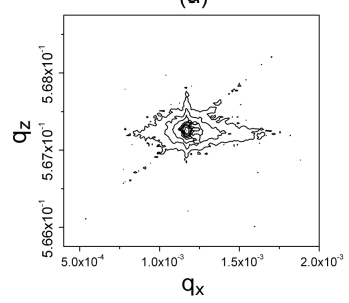

(b)

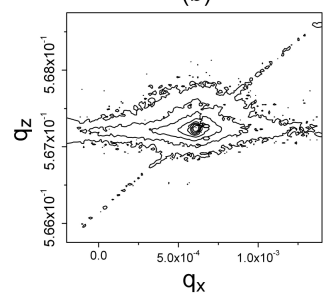

Fig. 3. 004 RSMs for Si:Mn at the second azimuthal position - after rotation for $90^{\circ}$, processed at 1270 $\mathrm{K}$ under $10^{5} \mathrm{~Pa}$ : (a) implanted at $340 \mathrm{~K}$ (b) implanted at $610 \mathrm{~K}$.

In the case of second other azimuthal position (after rotation for $90^{\circ}$ ), most $\mathrm{Si}: \mathrm{Mn}$ samples implanted at $340 \mathrm{~K}$ indicate the asymmetric distribution of diffracted X-ray intensity; the typical asymmetric distribution of X-ray intensity is presented in Fig. 3a. Such asymmetric intensity was observed for Si:Mn treated at $610 \mathrm{~K}$ under ambient and enhanced pressures, but, for the sample treated at $1270 \mathrm{~K}$, the asymmetric distribution was detected only after processing under $10^{5} \mathrm{~Pa}$. In the last case this effect was most pronounced. The presence of preferably oriented small crystallites in the implanted layer follows from the distribution of diffracted intensity changing with the azimuthal position. The lattice parameter of these crystallites is equal to that of the Si substrate.

For Mn:Si implanted at $610 \mathrm{~K}$, asymmetric X-ray diffuse scattering was detected after annealing at $1270 \mathrm{~K}$ under $10^{5} \mathrm{~Pa}$ only (Fig. $3 \mathrm{~b}$ ). Observed distribution of diffuse scattering increases for lower lattice parameter (larger $q_{z}$ ) indicating on a creation of interstitial defects [12]. 
(a)

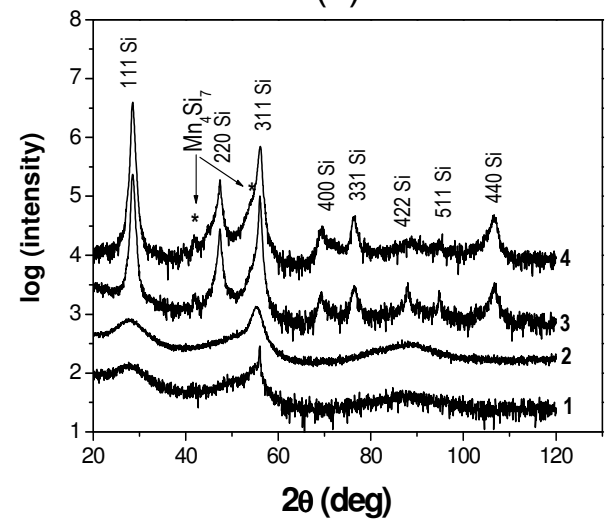

(b)

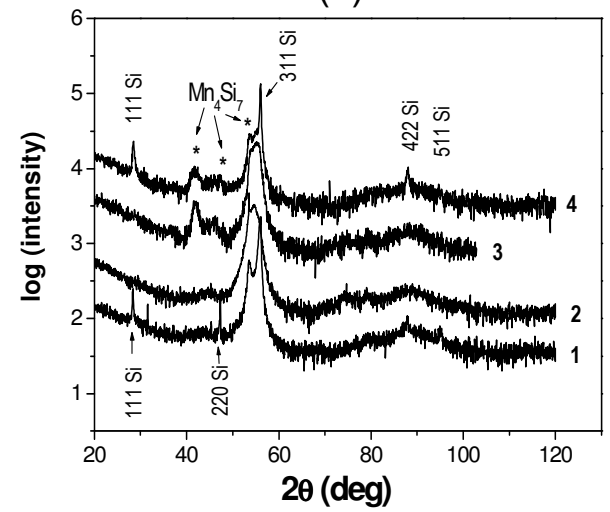

Fig. 4. Powder diffraction patterns in grazing incidence geometry for Si:Mn implanted at $340 \mathrm{~K}$ (a) and at $610 \mathrm{~K} \mathrm{(b):} 1$ - as-implanted; 2 - annealed for $1 \mathrm{~h}$ at $610 \mathrm{~K}$ under $10^{5} \mathrm{~Pa} ; 3,4$ - processed for $1 \mathrm{~h}$ at $870 \mathrm{~K}$ under $10^{5} \mathrm{~Pa}$ and $1.1 \mathrm{GPa}$, respectively.

$\mathrm{X}$-ray powder diffraction patterns in the grazing incidence geometry for Si:Mn implanted at 340 $\mathrm{K}$ and $610 \mathrm{~K}$ are presented in Fig. 4. For the sample implanted at $340 \mathrm{~K}$, the implanted layer remains to be amorphous both after implantation and after processing at $610 \mathrm{~K}$ (Fig. 4a). The reflections from polycrystalline $\mathrm{Si}$ were detected in the case of treatment under $1.1 \mathrm{GPa}$, showing on re-crystallisation of amorphous layer (Fig. 4a). In the case of implantation at $610 \mathrm{~K}$, the nearsurface layer in as-implanted Si:Mn is partially re-crystallised so the reflections coming from the $\mathrm{Si}_{7} \mathrm{Mn}_{4}$ phase are observed (Fig. 4b).

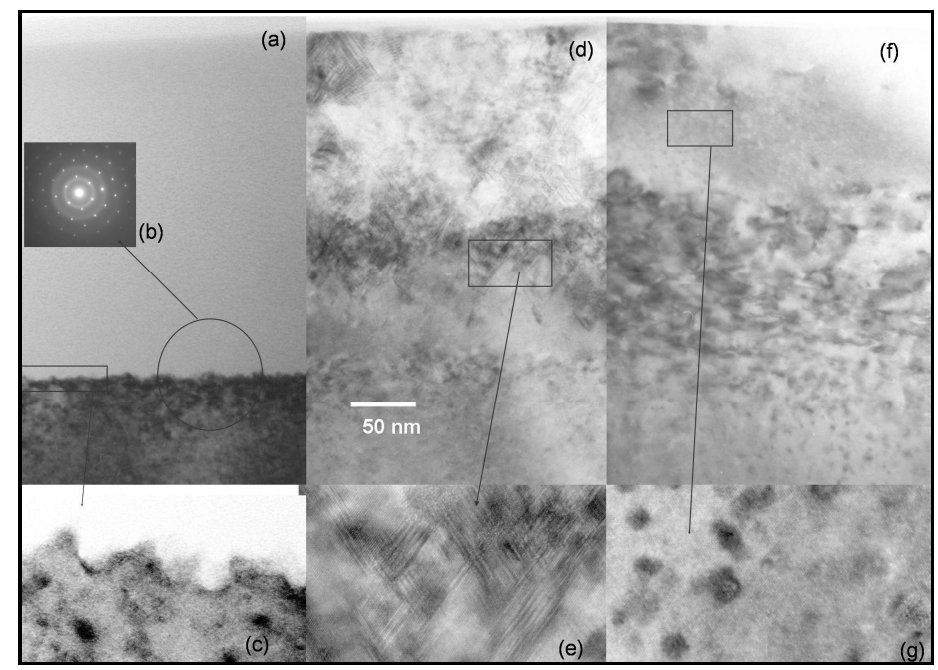

Fig. 5. TEM images: Si:Mn implanted at $340 \mathrm{~K}$ and next processed for $1 \mathrm{~h}$ at $610 \mathrm{~K}$ (a); its corresponding diffraction pattern (b); enlarged image of the amorphous/crystalline interface showing high surface roughness (c); Si:Mn processed at $870 \mathrm{~K}$ for $1 \mathrm{~h} \mathrm{(d);} \mathrm{planar} \mathrm{defects} \mathrm{at} \mathrm{the}$ middle part of the re-crystallized layer (e); Si:Mn implanted at $610 \mathrm{~K}$ and processed for $1 \mathrm{~h}$ at 870 $\mathrm{K}(\mathrm{f})$; precipitates of 3-6 $\mathrm{nm}$ dimension at upper part of Si:Mn (g).

After processing at $610 \mathrm{~K}$ re-crystallization of layer was only partial. Processing at $870 \mathrm{~K}$ results in the increased intensity of reflection coming from the $\mathrm{Si}_{7} \mathrm{Mn}_{4}$ phase. HP applied during processing promotes re-crystallisation of implantation - damaged material as results from observation of reflections originating from polycrystalline $\mathrm{Si}$ (Fig. $4 \mathrm{~b}$ ). The lattice parameters of the $\mathrm{Si}_{7} \mathrm{Mn}_{4}$ phase 
are as follows: $a=5.525 \AA$ and $c=17.463 \AA$ [14]. The defect structure changes caused by processing are probably responsible for magnetic properties reported for such samples $[10,11]$.

TEM images of Si:Mn implanted at $340 \mathrm{~K}$ and $610 \mathrm{~K}$ are presented in Fig. 5. The different defect structures of Si:Mn implanted at $340 \mathrm{~K}$ and $610 \mathrm{~K}$, and subsequently processed for $1 \mathrm{~h}$ at 870 $\mathrm{K}$ under $10^{5} \mathrm{~Pa}$, are distinctly detectable. In effect of processing at $870 \mathrm{~K}$, the planar defects (for example stacking faults with dislocation loops) are created in the sample implanted at $340 \mathrm{~K}$, while the same treatment of Si:Mn implanted at $610 \mathrm{~K}$ results in a creation of precipitates.

\section{Conclusions}

The high temperature-pressure treatment of Si:Mn modifies markedly its structure. The defect structure of processed samples depends first of all on the substrate temperature during implantation. The layered structure ceases to exist after processing of Si:Mn at $1270 \mathrm{~K}$, both under ambient and high pressures; dislocation loops (stacking faults) are created. At lower temperature $(870 \mathrm{~K})$ planar defects are created only in the case of Si:Mn prepared by implantation at $340 \mathrm{~K}$. Processing at 1270 $\mathrm{K}$ under $10^{5} \mathrm{~Pa}$ of $\mathrm{Si}: \mathrm{Mn}$ prepared by implantation at $340 \mathrm{~K}$ promotes most effectively a creation of $\mathrm{Si}$ precipitates with the preferred orientation. The creation of interstitials is most pronounced in Si:Mn prepared by implantation at $610 \mathrm{~K}$.

The tetragonal $\mathrm{Mn}_{4} \mathrm{Si}_{7}$ phase has been formed in processed $\mathrm{Si}: \mathrm{Mn}$; the $a$ and $c$ lattice parameters of tetragonal $\mathrm{Mn}_{4} \mathrm{Si}_{7}$ have been determined. More pronounced re-crystallization of amorphous material is observed for $\mathrm{Si}: \mathrm{Mn}$ processed under enhanced hydrostatic pressure.

\section{References}

[1] J. Kossut, W. Dobrowolski, in: Handbook of Magnetic Materials, ed. K.H.J. Buschow, North Holland, Amsterdam (1993), p. 231.

[2] H. Ohno, Science, 281 (1998), p. 951.

[3] F. Matsukura, H. Ohno, A. Shen, Y. Sugarawa, Phys. Rev. B, 57 (1998), p. R2037.

[4] T. Dietl, H. Ohno, F. Matsukura, J. Cibert, D. Ferrand, Science, 287 (2000), p. 1019.

[5] M. Zajac, J. Gosk, M. Kaminska, A. Twardowski, J. Szyszko, S. Podsiadlo, Appl. Phys. Lett., 79 (2001), p. 2432.

[6] M. Moreno, A. Trampert, B. Jenichen, L. Daweritz, K. Ploog, J. Appl. Phys., 92 (2002), p. 4672.

[7] H. Nakayama, H. Ohta, E. Kulatov, Phys. B, 302-303 (2001), p. 419.

[8] M. Bolduc, C. Awo-Affouda, A. Stollenwerk, M.B. Huang, F.G. Ramos, G. Agnello, V.P. LaBella, Phys. Rev. B, 71 (2005), p. 033302.

[9] Shegqiang Zhou, K. Potzger, Gufei Zhang, A. Mucklich, F. Eichhorn, N. Schell, R. Grotzschel, B. Schmidt, W. Skorupa, M. Helm, J. Fassbender, D. Geiger, Phys. Rev. B, 75 (2007), p. 0852003.

[10] A. Misiuk, J. Bak-Misiuk, B. Surma, W. Osinniy, M. Szot, T. Story, J. Jagielski, J. Alloys Comp., 423 (2006), p. 201

[11] A. Misiuk, B. Surma, J. Bak-Misiuk, Solid State Phen., 9 (2006), p. 270.

[12] M. Moreno, B. Jenichen, V. Kaganer, W. Braun, A. Trampert, L. Daweritz, K.H. Ploog, Phys. Rev. B, 67 (2003), p. 235206.

[13] U. Pietch, V. Holy, T. Baubach, in: High Resolution X-ray Scattering from Thin Film and Multilayers, Springer (2004), p. 170.

[14] JCPDS 72-2069 (International Committee for Diffraction Data 2002). 